\title{
AVALIAÇÃO DAS HABILIDADES ESCOLARES DE CRIANÇAS COM IMPLANTE COCLEAR
}

\section{Assessing skills of school children with cochlear implant}

\author{
Ana Beatriz Sacomano Montassier Pinheiro ${ }^{(1)}$, Midori Otake Yamada ${ }^{(2)}$, \\ Maria Cecília Bevilacqua ${ }^{(3)}$, Patrícia Abreu Pinheiro Crenitte ${ }^{(4)}$
}

\begin{abstract}
RESUMO
Objetivo: avaliar e caracterizar o desempenho escolar de crianças com deficiência auditiva usuárias de implante coclear, entre 9 e 12 anos, assim como, verificar o tempo que a criança leva para acessar a informação fonológica e confrontar estes resultados de acordo com o gênero e série dos participantes. Método: participaram deste estudo 32 crianças, de ambos os gêneros, freqüentando da primeira a quinta séries do ensino fundamental, regularmente matriculadas no Centro de Pesquisas Audiológicas do Hospital de Reabilitação de Anomalias Craniofaciais, Universidade de São Paulo, na cidade de Bauru/SP. Os instrumentos utilizados foram o Teste de Desempenho Escolar (TDE) e o Teste de Nomeação Automática Rápida (RAN). Resultados: os resultados mostraram que 74\% das crianças apresentaram desempenho escolar geral abaixo do esperado, com maior dificuldade na escrita e dentro da média para o tempo que levam ao processar a informação. Conclusões: concluiu-se que $74 \%$ das crianças apresentaram desempenho escolar inferior nas avaliações e que não houve relação entre estes resultados com a nomeação rápida, já que este está dentro da média e é um pré-requisito para a leitura, e os participantes deste estudo apresentam habilidade satisfatória para a leitura.
\end{abstract}

DESCRITORES: Criança; Implante Coclear; Educação

\section{INTRODUÇÃO}

A deficiência auditiva definida, como qualquer distúrbio no processo de audição normal, gera conseqüências amplas e devastadoras na vida de

(1) Psicóloga; Especialista em Psicologia Clínica e Hospitalar pelo Hospital de Reabilitação de Anomalias Craniofaciais da Universidade de São Paulo, Bauru/SP/Brasil.

(2) Psicóloga do Hospital de Reabilitação de Anomalias Craniofaciais da Universidade de São Paulo, Bauru-SP; Doutoranda em Psicologia da Faculdade de Filosofia Ciências e Letras de Ribeirão Preto, USP/SP/BR.

(3) Fonoaudióloga; Professora Titular do Departamento de Fonoaudiologia da Faculdade de Odontologia de Bauru da Universidade de São Paulo; Responsável pelo Centro de Pesquisas Audiológicas do Hospital de Reabilitação de Anomalias Craniofaciais da Universidade de São Paulo, Bauru/SP/BR.

(4) Fonoaudióloga; Professora do Departamento de Fonoaudiologia da Faculdade de Odontologia de Bauru da Universidade de São Paulo, Brasil; Doutora em Ciências Médicas pelo Departamento de Neurologia da Faculdade de Ciências Médicas da Universidade de Campinas.

Conflito de interesses: inexistente uma criança, sobretudo quando diagnosticada de graus severo ou profundo ${ }^{1}$. Nos primeiros anos de vida, essa privação sensorial impede ou dificulta a aquisição da linguagem oral, originando dificuldades no convívio familiar e social, no desempenho acadêmico, e na fase adulta podendo surgir dificuldades na profissionalização ${ }^{2,3}$.

Estudos $^{4}$ realizados com deficientes auditivos, alunos do ensino médio ou cursando ensino superior, que pretendiam delimitar as facilidades e dificuldades para chegar ao ensino superior, constataram o baixo acesso destes indivíduos a melhor oportunidade de estudo e, conseqüente, empregos qualificados. As autoras apontam como fator diferencial a importância da reabilitação do deficiente auditivo e atenção especial na fase pré-escolar, fase em que a criança adquire a base para chegar ao ensino superior.

Diante dos avanços tecnológicos no mundo, o implante coclear (IC) é, atualmente, um efetivo recurso clínico no tratamento de indivíduos com deficiência auditiva, por garantir melhora da qualidade 
de vida do deficiente auditivo neurossensorial bilateral de graus severo e profundo 5 . O IC possibilita a habilitação de crianças deficientes auditivas que não se beneficiam com os aparelhos de amplificação sonora individuais (AASI), no desenvolvimento de habilidades auditivas ${ }^{6}$. Assim, provê a sensação da audição à criança deficiente auditiva com a qualidade necessária para a percepção dos sons da fala. A idade em que as crianças são implantadas, o tempo de privação sensorial, o envolvimento da família, as características individuais da criança e o acompanhamento pós-cirúrgico rigoroso são alguns fatores que contribuem para o sucesso do implante $^{5,7,8,9,10}$.

Tem-se observado um aumento gradativo de usuários de implante coclear nos mais diversos contextos em que vivemos. E não diferente, as escolas estão, cada vez mais, recebendo crianças com implante coclear. Contudo, faltam informações referentes ao implante coclear para a população em geral, em especial para as escolas, que podem receber com insegurança seus alunos por desconhecimento sobre a criança deficiente auditiva usuária de implante coclear. Existe também uma falsa idéia de que as eventuais dificuldades enfrentadas por uma criança com deficiência auditiva na escola estarão solucionadas com a utilização do implante coclear.

Vários estudos apontam a idade em que estas crianças devem receber o implante como um dos principais fatores de sucesso da reabilitação. Sugerem que deve ser realizado o mais cedo possível, preferencialmente primeiro ano de vida $^{11-13}$. Embora tenham acesso eficaz às informações acústicas da língua portuguesa e benefícios em seu desenvolvimento de linguagem oral após o uso efetivo do implante coclear e reabilitação adequada, as crianças com implante coclear ainda podem sofrer algumas desvantagens de privação sensorial auditiva para o desenvolvimento infantil. Estudos indicam que as crianças com implante coclear se beneficiam em explicações individuais de seus professores, mas apresentam dificuldades quando as explicações são para o grupo ${ }^{14}$. Outros reafirmam a necessidade de suportes educativos adicionais para o bom desempenho educacional. Ainda assim, são poucos os estudos no meio científico sobre o desempenho escolar de crianças com implante coclear ${ }^{15}$.

O que não se tem muito conhecimento, pelo menos no Brasil, é como os professores estão lidando com esta nova demanda e como estão se desenvolvendo estas crianças, principalmente em suas habilidades de leitura e escrita. Sobre este aspecto um estudo brasileiro analisou o desempenho escolar de cinco crianças deficientes auditivas usuários de implante coclear, matriculadas na $3^{\underline{a}}$ série do ensino regular, em escola particular e mostrou que apenas uma apresentou bom desempenho escolar, enquanto as outras três apresentaram poucas dificuldades e uma apresentou muita dificuldade para acompanhar o ensino ${ }^{16}$. Em outro estudo, as alterações ortográficas de crianças com implante coclear também foram analisadas e concluiu-se que cometeram erros em alto índice ${ }^{17}$. $\mathrm{E}$, especificamente sobre o desempenho escolar de crianças usuárias de implante coclear uma pesquisa com 70 crianças apresentou como resultado um desempenho abaixo do esperado para a série frequentada, porém conclui que as crianças usuárias de implante coclear evoluem a cada série cursada e afirma que as crianças possuem benefícios em classes comuns no ensino regular ${ }^{18}$.

Em suma, estes estudos mostram que existe melhoria significativa na compreensão e nas habilidades de leitura e aprendizagem para as crianças usuárias de implante coclear, além da melhora das habilidades auditivas e de linguagem, como são vistos na maioria dos estudos. Questões como leitura, escrita e aritmética são sempre exigidas pelos adultos, pais e professores, que esperam ser desenvolvidas nas crianças. E diante dessa situação, buscamos algumas respostas, por meio de instrumentos específicos, a fim de investigar mais sobre a situação da criança com deficiência auditiva, usuária de implante coclear no meio acadêmico.

Portanto, o objetivo deste estudo, foi avaliar e caracterizar o desempenho escolar de crianças com deficiência auditiva usuárias de implante coclear, entre 9 e 12 anos, assim como, verificar o tempo que a criança leva para acessar a informação fonológica e confrontar estes resultados de acordo com o gênero, verificar se há uma relação entre tempo de implante coclear com o desempenho na leitura,escrita,e aritmética.

\section{MÉTODO}

A seleção dos sujeitos foi realizada aleatoriamente de acordo com a data da de rotina de atendimento das crianças implantadas, previamente agendadas pelo setor. Foi utilizado o atendimento psicológico para a aplicação dos instrumentos para a coleta dos dados, sendo, portanto, uma sessão de aproximadamente uma hora cada criança.

Participaram deste estudo 32 crianças deficientes auditivas pré-linguais, usuárias de implante coclear, entre 9 e 12 anos de idade, regularmente matriculadas no CPA/HRAC-USP. 
Os instrumentos utilizados foram:

a) Teste de Desempenho Escolar (TDE) - elaborado para avaliar as habilidades acadêmicas básicas de escolares de $1^{\underline{a}}$ a $6^{\underline{a}}$ séries do ensino fundamental, relacionadas à leitura, escrita e aritmética, por séries, classificando o desempenho em inferior, médio e superior.

$O$ teste indica quais as áreas da aprendizagem escolar estão prejudicadas ou preservadas no avaliando e é composto por três subtestes: 1 . Escrita (escrita do nome próprio e de palavras isoladas sob a forma de ditado); 2. Aritmética (solução oral de problemas e cálculo de operações aritméticas por escrito); e, 3. Leitura (reconhecimento de palavras isoladas do contexto) ${ }^{19}$.

b) Teste de Nomeação Automática Rápida (RAN) - instrumento que mede a velocidade do analisando em acessar e recuperar atividades verbais na nomeação contínua de diversos estímulos visuais e utilizado para diagnosticar problemas potenciais da leitura, permitindo a detecção precoce e a devida intervenção. É composto por quatro conjunto de estímulos para nomeação: cores (vermelho, amarelo, verde, azul, preto), dígitos (2, 4, 6, 7, 9), letras minúsculas ( $\mathrm{a}, \mathrm{d}, \mathrm{o}, \mathrm{s}, \mathrm{p})$ e desenhos de objetos comuns (guarda-chuva, tesoura, pente, relógio, chave) ${ }^{20}$.

Esse estudo foi realizado no Centro de Pesquisas Audiológicas (CPA) do Hospital de Reabilitação de Anomalias Craniofaciais da Universidade de São Paulo (HRAC-USP), na cidade de Bauru/SP e aprovado conforme ofício no 94/2010-SVAPEPE-CEP.

Os resultados deste estudo foram submetidos a uma análise quantitativa, qualitativa e comparativa, verificando o nível de significância das diferenças encontradas no desempenho de cada criança. Nesta análise estatística comparativa, foram utilizados os testes Mann-Whitney e o Coeficiente de Correlação de Spearman. Para os dois testes estatísticos foi utilizado o nível de significância $p<0,05$.

\section{RESULTADOS}

Participaram deste estudo 32 crianças com deficiência auditiva usuárias de implante coclear, sendo $20(62 \%)$ do gênero feminino e 12 (38\%) do gênero masculino. A faixa etária dos participantes continuou entre 9 e 12 anos, porém a maioria estava com 09 anos de idade no momento da coleta de dados, representando $40 \%$ das crianças, seguido de 10 anos (25\%), 11 anos (22\%) e 12 anos (13\%).

De acordo com os dados obtidos pelo responsável da criança, presente no momento da coleta de dados, a maioria das crianças freqüentava a $3^{\text {a }}$ série, representando $41 \%$, seguida da $4^{\text {a }}$ série $(25 \%)$, da $2^{\underline{a}}$ série $(19 \%)$, da $5^{\text {a }}$ série $(9 \%)$ e da $1^{\underline{a}}$ série $(6 \%)$.

Com relação ao tempo de implante, a média de idade na época da cirurgia foi de 3 anos e 6 meses (de 1 ano e 10 meses a 6 anos). Apenas duas crianças realizaram a cirurgia com menos de 2 anos de idade, representando $6 \%$ dos participantes.

A maioria das crianças, ou seja, $35 \%$ realizaram a cirurgia com idade entre 2 anos e 1 mês à 3 anos, seguido de $34 \%$ de crianças que realizaram a cirurgia com idade entre 3 anos e 1 mês a 4 anos. Os demais realizaram a cirurgia com idade entre 4 anos e 1 mês e 5 anos (16\%) e entre 5 anos e 1 mês e 6 anos (9\%).

Nota-se que não há um padrão entre a idade e a série frequentadas, pois a maioria das crianças avaliadas que possuem 9 anos de idade frequentam tanto a $3^{\text {a }}$ quanto a $2^{\underline{a}}$ ou até a $1^{\text {a }}$ série. $O$ mesmo ocorre nas idades de 10, 11 e 12 anos: as crianças com 10 anos frequentam tanto a $4^{\text {a }}$ como a $3^{\text {a }}$ séries; as crianças com 11 anos freqüentam a $4^{\text {a }}$ e $5^{\mathrm{a}}$ séries e as de 12 anos freqüentam a $4^{\mathrm{a}}$ e a $3^{\mathrm{a}}$ séries.

Quanto ao desempenho acadêmico geral das crianças deste estudo, de acordo com o proposto pelo TDE, é possível observar na Figura 1 que a maioria, ou seja, $74 \%$ apresentaram desempenho inferior nas avaliações, seguidas das crianças que apresentaram desempenho superior e dentro da média, sendo $13 \%$ em cada categoria $(p=<0,001$, $\mathrm{dp}=0,80$ ).

$24 ; 74 \%$

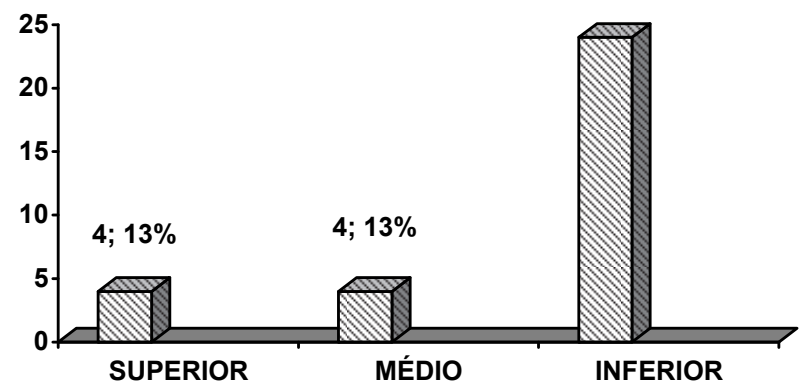

Figura 1 - Desempenho geral dos participantes no TDE

$\mathrm{Na}$ Figura 2, observa-se a classificação de desempenho de acordo com o TDE quanto às áreas de escrita, aritmética e leitura. Neste estudo, conforme os resultados obtidos, nota-se que a área da escrita foi a de maior dificuldade para as crianças $(81 \%)$, seguida da leitura $(72 \%)$ e da aritmética $(47 \%)$. 


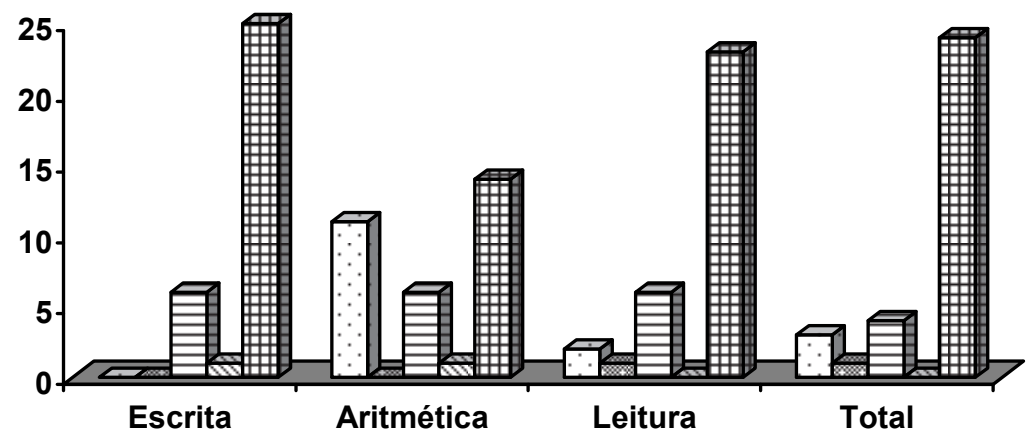

口Superior 四 Médio Superior 曰Médio $⿴$ Médio Inferior

Figura 2 - Distribuição de acordo com a classificação de desempenho no TDE

Na Figura 3, é possível observar os resultados da classificação de desempenho de acordo com o proposto pelo RAN, quanto ao tempo de nomeação de cores, dígitos, letras e objetos. Assim, verifica-se que a $53 \%$ das crianças apresentou desempenho acima da média para a nomeação rápida de dígitos e $56 \%$ desempenho acima da média para letras, $66 \%$ das crianças apresentaram desempenho dentro da média para a nomeação de cores, e $69 \%$ abaixo da média para a nomeação de objetos.

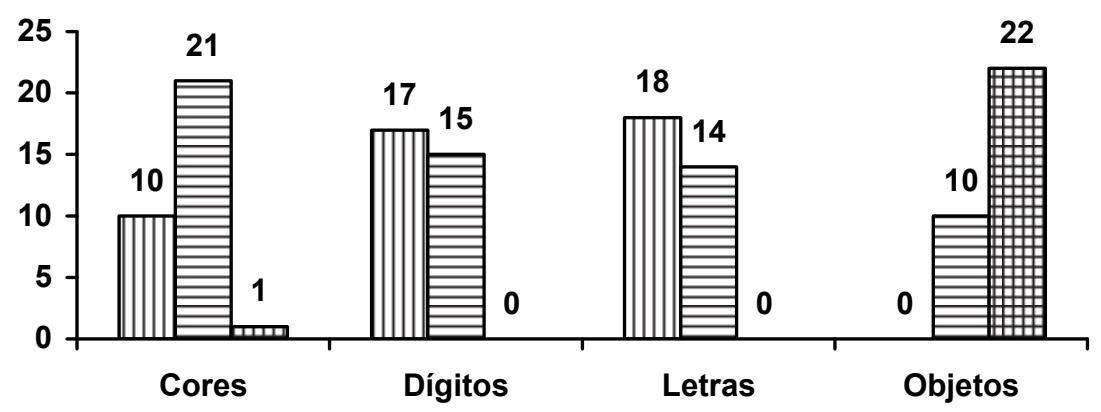

凹 Acima da média $\quad$ Média $\boxplus$ Abaixo da média

Figura 3 - Distribuição de acordo com a classificação de desempenho no RAN

Quanto à comparação estatística de acordo com o gênero em ambos os testes TDE e RAN, obtemos um resultado significativo somente na tarefa de aritmética do TDE $(U=69,5$ e $p=0,048)$, sendo que as meninas apresentaram desempenho melhor do que os meninos, conforme a Tabela 1.

Realizando a correlação estatística entre os testes TDE e RAN, como resultado significativo observa-se, na Tabela 2, a discrepância no desempenho das crianças avaliadas no TDE entre as provas de escrita e leitura $(R=0,50$ e $p=0,004)$ e entre aritmética e leitura $(R=0,36$ e $p=0,041)$. No teste RAN, os resultados significativos foram para o tempo de nomeação rápida entre cores e dígitos
$(R=0,39$ e $p=0,028)$ e entre cores e objetos $(R=$ $0,42$ e $p=0,016)$.

Com relação ao tempo de implante, a média de idade na época da cirurgia foi de 3 anos e 6 meses (de 1 ano e 10 meses a 6 anos). Apenas duas crianças realizaram a cirurgia com menos de 2 anos de idade, representando $6 \%$ dos participantes.

A maioria das crianças, ou seja, $35 \%$ realizaram a cirurgia com idade entre 2 anos e 1 mês à 3 anos, seguido de $34 \%$ de crianças que realizaram a cirurgia com idade entre 3 anos e 1 mês à a 4 anos. Os demais realizaram a cirurgia com idade entre 4 anos e 1 mês e 5 anos (16\%) e entre 5 anos e 1 mês e 6 anos $A$ idade de implantação das crianças 
Tabela 1 - Comparação entre os gêneros no desempenho do TDE e do RAN

\begin{tabular}{lcccc}
\hline \multicolumn{1}{c}{ Provas } & Fem & Masc & U & Valor de $\mathbf{~ p}^{\text {* }}$ \\
\hline TDE - Escrita & 339 & 189 & 111 & 0,744 \\
TDE - Aritmética & 279,5 & 248,5 & 69,5 & 0,048 \\
TDE - Leitura & 335,5 & 192,5 & 114,5 & 0,833 \\
TDE - Geral & 322 & 206 & 112 & 0,774 \\
\hline RAN - Cores & 306,5 & 221,5 & 96,5 & 0,366 \\
RAN - Dígitos & 352 & 176 & 98 & 0,408 \\
RAN - Letras & 358 & 170 & 92 & 0,289 \\
RAN - Objetos & 310 & 218 & 100 & 0,454 \\
\hline
\end{tabular}

*valor de $p$ considerado: $p<0,05$

Tabela 2 - Correlação entre todas as escalas dos intrumentos TDE E RAN

\begin{tabular}{llcc}
\hline \multicolumn{1}{c}{ Provas } & N & R & Valor de $\mathbf{~ p}^{\mathbf{*}}$ \\
\hline TDE Escrita X TDE Leitura & 32 & 0,50 & 0,004 \\
TDE Leitura X TDE Escrita & 32 & 0,50 & 0,004 \\
TDE Aritmética X TDE Leitura & 32 & 0,36 & 0,041 \\
TDE Leitura X TDE Aritmética & 32 & 0,36 & 0,041 \\
\hline RAN Cores X RAN Dígitos & 32 & 0,39 & 0,028 \\
RAN Dígitos X RAN Cores & 32 & 0,39 & 0,028 \\
RAN Cores X RAN Objetos & 32 & 0,42 & 0,016 \\
RAN Objetos X RAN Cores & 32 & 0,42 & 0,016 \\
\hline
\end{tabular}

*valor de $p$ considerado: $p<0,05$

parece ser um forte influenciador para o seu desempenho em desenvolvimento geral, atuando no prognóstico do seu desempenho acadêmico embora não tenha se observado associações entre os desempenhos das crianças nos testes e o tempo de privação sensorial.

Foi possível observar também nas análises do presente estudo que as crianças que receberam o implante coclear até os 3 anos e meio de idade aproximadamente, obtiveram resultados mais favoráveis em relação ao seu desempeno acadêmico.

\section{DISCUSSÃO}

Os benefícios do implante coclear quanto à linguagem e à comunicação em crianças com deficiência auditiva tem sido relatado na literatura ${ }^{2,5}$ como pontuaram anteriormente. Entretanto, no que se refere às habilidades escolares de crianças, especialmente sobre a leitura e escrita, usuárias de implante coclear a literatura nacional atualmente é bastante escassa.

É por meio da audição que o indivíduo pode compreender a linguagem oral, formar conceitos, interrelacioná-los e posteriormente expressá-los através da fala, tendo capacidade articulatória para isto ${ }^{2}$. Desse modo, fica evidente a importância da audição para o desenvolvimento da linguagem oral e seu prejuízo, interferindo na comunicação oral como um todo. Diante disso, autores pontuam sobre a contribuição do implante coclear na aquisição da linguagem oral afirmando que este dispositivo possibilita o acesso aos sons da fala e, assim, o desenvolvimento das habilidades auditivas em etapas gradativas ${ }^{21}$.

Entretanto, o principal efeito da deficiência auditiva está na repercussão do desenvolvimento da linguagem e da fala, o que, consequentemente, interfere em todo o processo de aprendizagem e desempenho acadêmico' ${ }^{1}$.

Sendo assim, neste estudo observou-se que quanto ao desempenho geral das crianças com deficiência auditiva, usuárias de implante coclear, de acordo com o proposto pelo TDE, a maioria, ou seja, $74 \%$ apresentaram desempenho inferior nas avaliações, seguida das crianças que apresentaram desempenho superior e dentro da média, sendo $13 \%$ em cada categoria. 
Em um estudo semelhante ${ }^{8}$, o desempenho de crianças com implante coclear, na faixa etária entre 6 e 12 anos, evidenciou que $62,5 \%$ dos pais entrevistados na pesquisa relataram que seus filhos apresentam dificuldades escolares, em especial na aprendizagem da leitura e escrita e acompanhamento das atividades de sala de aula como seus colegas ouvintes.

Ainda evidenciando o desempenho acadêmico das crianças com implante coclear, cinco crianças usuárias de implante coclear, entre 9 e 12 anos, foram analisadas especificamente nas provas de Português e Matemática e concluíram que apenas uma apresentou bom desempenho e que as dificuldades foram evidentes, especialmente em Português ${ }^{16}$.

Em outro estudo, a ortografia de crianças usuárias de implante coclear foi avaliada e constatou-se que a maioria das crianças erraram as atividades propostas trocando fonemas surdos (/f/) e sonoros (/v/), seguidos dos erros em omissões de letras. De acordo com este caso, bem como deste estudo, na surdez neurossensorial as primeiras células ciliadas a serem lesadas estão na base da cóclea, onde ocorre a vibração das freqüências agudas e onde a perda auditiva tende a ser mais profunda ${ }^{17}$. Assim foi possível entender as alterações na escrita quanto à relação fonemas/grafemas, localizados em freqüências agudas por seu aspecto anatômico e funcional, o que confirma os achados deste estudo que revelou que 25 , das 32 crianças avaliadas apresentaram dificuldades na escrita.

Mais um estudo que confirma nossos achados foi a pesquisa que avaliou o desempenho educacional de crianças com implante coclear e mostrou uma porcentagem significativa de crianças classificadas abaixo do nível médio ${ }^{22}$.

Nesta perspectiva, outros achados relatam que a compreensão da leitura entre as crianças usuárias de implante coclear é significativamente melhor do que a de crianças com AASI, porém, ainda muito atrás a de crianças ouvintes. $O$ implante coclear foi associado com a vantagem no reconhecimento da palavra quando comparado com o usuário do AASI, isso com os alunos do ensino médio, não com os alunos do ensino fundamental ${ }^{23}$.

Como instrumento facilitador para o desenvolvimento da interpretação e compreensão de textos pelos deficientes auditivos, a dramatização é apresentada como possibilidade de trabalhar as dificuldades para a compreensão do texto escrito, principalmente pela falta de domínio da linguagem oral porque são prejudicados durante o período de desenvolvimento da linguagem ${ }^{24}$.

Os surdos são privados da compreensão de diálogos com palavras usuais do repertório dos ouvintes e, em consequência do sistema conceitual do grupo sociocultural predominante do qual participam, não internalizam muito dos conceitos, sobretudo os abstratos e, com isso, não dominam de forma satisfatória a função planejadora e organizadora da linguagem ${ }^{24}$.

Quanto ao desempenho acadêmico observou-se um baixo desempenho da escrita quando comparado com o desempenho em leitura e aritmética.

Foi observado um baixo desempenho da escrita quando comparado com o desempenho em leitura e aritmética. Com relação ao tempo de implante, pesquisas afirmam que as crianças que eram mais jovens quando receberam o implante tendem a ter uma melhor pontuação para a compreensão de leitura e demonstram melhores competências linguísticas. Também apontam que o status socioeconômico influencia para a compreensão da leitura: quanto mais baixo, pior o rendimento da leitura ${ }^{25}$.

Tais estudos confirmam os achados desta pesquisa com relação ao desempenho para a compreensão da leitura, visto que a maioria das crianças que realizaram a cirurgia mais precocemente, ou seja, em torno de seus 2 anos e meio de idade, apresentaram bom desempenho na leitura.

No entanto, quando avaliado o desenvolvimento da fala, linguagem, leitura e habilidades escolares de crianças implantadas, inicialmente entre 8 e 9 anos, até completarem os 15 e 18 anos, observa-se ganhos significativos em com a utilização do implante coclear, com exceção da leitura que não chegou a bons níveis no ensino médio para a maioria dos estudantes ${ }^{26}$.

Afirma-se que o implante coclear proporciona potencial para a construção da linguagem através de situações naturais do dia a dia, usando a audição como fonte primária para o desenvolvimento da linguagem sendo um recurso efetivo para a criança que recebe esta tecnologia nos primeiros anos de vida $^{5}$.

Quanto à diferença encontrada entre os gêneros feminino e masculino, os resultados desta pesquisa, referentes ao subteste de aritmética no TDE, corroboram com os de um estudo em que se comparou o desempenho acadêmico de um grupo de escolares com desempenho normal com de outro grupo abaixo da média, segundo a opinião dos professores. Como resultado, verificou-se uma diferença significativa entre os dois grupos, indicando que o desempenho inferior foi verificado para o gênero masculino e para a quarta série, confirmando a opinião dos professores quanto aos grupos ${ }^{27}$.

Outro estudo pontua que o baixo rendimento acadêmico é mais comum entre meninos que entre meninas, bem como a alta taxa de problemas na leitura e quando se referem à dificuldade de 
aprendizagem. Seja menino ou menina, sugere dar atenção às dificuldades emocionais e comportamentais de cada criança ${ }^{28}$, assim como nos conflitos das relações familiares, verificados nos atendimentos clínicos realizados.

A literatura sobre a nomeação automática rápida descrita diverge quanto à sua vinculação ao processamento fonológico ou a velocidade no processamento da informação. O processamento fonológico é explicado pela informação fonológica recebida auditivamente, estando diretamente ligada ao desenvolvimento da linguagem oral e escrita, sendo composto pela consciência fonológica, memória fonológica e a nomeação automática rápida ${ }^{29}$.

É importante saber que a capacidade da criança implantada escutar os sons da fala não significa que ela consiga processar tais informações complexas auditivamente. Desse modo, vê-se a importância em habilitar professores e outros profissionais para garantir a melhor comunicação e compreensão da mensagem pela criança em sala de aula ${ }^{30}$.

Utilizando a prova de nomeação rápida (RAN), um estudo realizado com crianças entre 8 e 12 anos apontou que as diagnosticadas com transtorno do déficit de atenção e hiperatividade (TDAH) e dislexia (transtorno da leitura e da escrita) apresentaram desempenho inferior ao de crianças que lêem conforme o esperado para esta idade e escolaridade, demonstrando que tal habilidade pode ser considerada como um pré-requisito para o desempenho em leitura ${ }^{29}$.

De acordo com a literatura internacional, a nomeação rápida é considerada um indicador para o desenvolvimento da leitura e da escrita. Tais resultados não coincidem com nossos achados quanto à prova de nomeação rápida (RAN), pois observamos que a maioria das crianças apresentou desempenho acima da média para a nomeação rápida de dígitos e letras e desempenho dentro da média para a nomeação em cores e apresentaram desempenho abaixo da média para a nomeação de objetos. Para justificar tais achados pode se afirmar que nomear é um processo que inclui pelo menos três estágios essenciais: identificar o objeto, ativar seu nome e generalizar a resposta. Primeiro, um objeto deve ser identificado como sendo de uma classe particular de objetos, por sua aparência ou traçado, orientação e disposição. Então, nomes apropriados devem ser ativados entre as palavras já conhecidas no léxico mental e finalmente deve haver uma organização fonológico-articulatória para que uma resposta específica possa ser executada ${ }^{31}$.
Os testes que avaliam a habilidade de nomeação rápida requerem que as crianças codifiquem a informação visual (figuras, dígitos e letras), recuperem rótulos lexicais da memória de longo termo (por exemplo, os códigos fonológicos) e os articulem ${ }^{32}$.

Nota-se que os dois testes de maior complexidade verbal (nomeação de cores e objetos) têm perfomances inferiores aos de dígitos e letras. A nomeação do número "1" requer bem menos tempo que "guarda-chuva" e "amarelo".

Estudos afirmam que a nomeação é mais rápida para letras e números do que para cores, uma vez que a nomeação de estímulos requer uso de processos atencionais, perceptivos e visuais para recuperação de léxico de maior extensão, corroborando com os achados. Ainda é importante ressaltar que os efeitos de supressão articulatória e de extensão de palavras estão localizados no processo de rechamada subvocal, e que, se houver qualquer alteração no processamento lingüístico da informação, este inibirá o acesso ao estoque fonológico, gerando falhas para nomeação de palavras com maior extensão, como na nomeação de cores e objetos $^{33}$. Assim, podemos inferir que o bom desempenho nesta habilidade do processamento fonológico pode estar relacionado com o bom desempenho na leitura.

Um estudo que contribui com tais achados avaliou o desempenho de leitores e não-leitores em tarefas relacionadas a diversas habilidades liguístico-cognitivas, mostrando que os leitores apresentaram melhor desempenho, inclusive na prova de nomeação automática rápida (RAN), e concluiu que a experiência da leitura influencia neste bom resultado ${ }^{34}$.

Contudo, além do uso efetivo do implante coclear, sua funcionalidade depende da qualidade e da freqüência da intervenção terapêutica que sucede a implantação, pois as crianças que realizam a cirurgia de implante coclear devem ser auxiliadas a interpretar e dar significados aos sons que são transmitidos para o seu cérebro. Desse modo, aquelas que possuem acesso a um programa terapêutico têm melhor oportunidade de se beneficiar do implante coclear ${ }^{17}$, além disso, o apoio e envolvimento da família, parte importante deste processo, se torna cada vez mais indispensável.

Este estudo demonstra a seriedade da reabilitação precoce para o deficiente auditivo como tentativa de evitar conseqüências devastadoras da privação sensorial que nos primeiros anos de vida impede ou dificulta a aquisição da linguagem oral, originando dificuldades no convívio familiar e social, desempenho acadêmico, podendo surgir dificuldades, na fase adulta, para a profissionalização. 


\section{CONCLUSÃO}

Mediante avaliação realizada no presente estudo, conclui-se que o desempenho escolar geral das crianças deficientes auditivas usuárias de implante coclear, na faixa etária de 9 e 12 anos, foi abaixo do esperado.

Os instrumentos utilizados se mostraram eficientes para avaliar e caracterizar o desempenho escolar das crianças deficientes auditivas usuárias de implante coclear, permitindo sua caracterização neste estudo.

Com relação aos subtestes de leitura, escrita e aritmética, a maioria dos participantes avaliados apresentou melhor desempenho na aritmética, seguido da leitura e maior dificuldade na escrita.

Quanto ao desempenho das crianças na prova de nomeação rápida (RAN) verificado entre os participantes deste estudo verifica-se que a maioria está dentro da média nos subtestes cores, dígitos e letras, com exceção do subteste objetos.

Confrontando os resultados de acordo com o gênero, conclui-se que as meninas apresentaram melhor desempenho do que os meninos apenas no subteste de TDE-aritmética.

Pode-se inferir que não houve relação entre o baixo desempenho das crianças usuárias de implante coclear na prova TDE-leitura e TDE-escrita, com a prova de nomeação rápida (RAN), visto que os participantes deste estudo apresentaram desempenho satisfatório no RAN, considerado pelos estudiosos um pré-requisito para o bom desempenho da leitura e da escrita.

Com os resultados deste estudo, verifica-se a necessidade do apoio educacional às crianças deficientes auditivas usuárias de implante coclear aliado à adequada reabilitação. Destaca-se como sugestão para continuidade da pesquisa, a intervenção com os professores destas crianças, a fim de se obter melhora no seu desempenho escolar.

Tais resultados, portanto, contribuem para a comunidade científica, para os profissionais da área, pais e crianças usuárias do implante coclear, que, atuando juntos na elaboração de estratégias possam amenizar as dificuldades escolares das crianças usuárias de implante coclear.

\begin{abstract}
Purpose: to evaluate and characterize the performance of children with hearing impairment using cochlear implants, between 9 and 12 year old, as well as to check the time that the child takes to access phonological information and confront such results according to gender and number of participants. Method: the study included 32 children of both genders, attending the first to the fifth grade of elementary school, regularly enrolled in the Audiological Research Center of Rehabilitation Hospital of Craniofacial Anomalies, University of São Paulo, in Bauru / SP. The instruments used were the School Performance Test (TDE) and the Rapid Auto Naming Test (RAN). Results: the results showed that $74 \%$ of children showed school performance being generally below expected, with greater difficulty in writing and within the average as for the time it takes to process the information. Conclusions: we conclude that $74 \%$ of children had lower academic performance assessments and that there was no relationship among such results with the rapid naming, as this is within the mean value and is a prerequisite for reading, and the participants in this study provide satisfactory reading skill.
\end{abstract}

KEYWORDS: Child; Cochlear Implantation; Education

\section{REFERÊNCIAS}

1. Bevilacqua MC. Conceitos Básicos sobre Audição e Deficiência Auditiva. Bauru: Secretaria de Saúde do Estado de São Paulo; 1998.

2. Stuchi RF, Nascimento LT, Bevilacqua MC, Brito Neto RV. Linguagem oral de crianças com cinco anos de uso de implante coclear. Pró-Fono. 2007;19(2):167-76.
3. Moret AL. Princípios básicos da habilitação da criança deficiente auditiva com implante coclear. In: Bevilacqua MC, Moret ALM, organizadoras. Deficiência auditiva: conversando com familiares e profissionais de saúde. São José dos Campos: Pulso; 2005. p. 225-34.

4. Manente MV, Rodrigues OMPR, Palamin MEG. Deficientes auditivos e escolaridade: fatores diferenciais que possibilitam o acesso ao ensino superior. Rev Bras Educ Espec. 2007;13(1):27-42. 
5. Bevilacqua MC, Costa Filho OA, Martinho AC. Implante Coclear. In: Ferreira LP, Befi-Lopes DM, Limongi SC, organizadores. Tratado de Fonoaudiologia. São Paulo: Roca; 2004. p. 751-61.

6. Costa Filho OA, Bevilacqua MC. Implantes cocleares multicanais em crianças. In: Caldas Neto SC, Sih T. Otologia e Audiologia em Pediatria. Rio de Janeiro: Revinter; 1999. p. 266-73.

7. Moraes TV, Zeigelboim BS, Bevilacqua MC, Jacob LC. Indicação de implante coclear: tendências atuais. Acta Awho. 2001;20(4):229-37.

8. Bevilacqua MC, Costa Filho OA, Moret AL. Implante coclear em crianças. In: Campos CA, Costa $\mathrm{HO}$, editores. Tratado de otorrinolaringologia. São Paulo: Roca; 2002. p. 268-77.

9. Hellman SA et al. The development of a children's implant profile. Am Ann Deaf. 1991;136(2):77-81.

10. Bevilacqua MC, Costa OA, Amantini RB. Considerações sobre o implante coclear em crianças. In: Bevilacqua MC, Moret AL. Deficiência auditiva: conversando com familiares e profissionais da saúde. São José dos Campos: Pulso; 2005. p. 123-38.

11. Nikolopoulos TP, O'Donoghue GM, Archbold S. Age at implantation: its importance in pediatric cochlear implantation. Laryngoscope. 1999;109(4):595-9.

12. Miyamoto R, Houston DM, Kirk KI, Perdew AE, Svirsky MA. Language development in deaf infants following cochlear implantation. Acta Otolaryngol. 2003;123(2):241-4.

13. Moret AL, Bevilacqua MC, Costa Filho OA. Implante coclear: audição e linguagem em crianças deficientes auditivas pré-linguais. Pró-Fono. 2007;19(3):295-304.

14. Preisler $G$, Tvingstedt AL, Ahlström M. Interviews with deaf children about their experiences using cochlear implants. Am Ann Deaf. 2005;150(3):260-7.

15. Preisler G, Tvingstedt AL, Ahlström M. A psychosocial follow-up study of deaf preschool children using cochlear implants. Child Care Health Dev. 2002;28(5):403-18.

16. Gabriele DP, Bevilacqua MC. Crianças deficientes auditivas usuárias de implante coclear multicanal e o contexto escolar. Temas Desenvolv. 2007; 15:85-91.

17. Lemes JM. Análise da ortografia de crianças usuárias de implante coclear. [Dissertação]. Rio de Janeiro (RJ): Universidade Velga de Almelda; 2007.

18. Brazorotto JS. Desempenho escolar em crianças usuárias de implante coclear. [Tese]. São Carlos (SP): Centro de Educação e Ciências Humanas, Universidade Federal de São Carlos; 2008.
19. Stein LM. Teste de desempenho escolar: manual para aplicação e interpretação. São Paulo: Casa do Psicólogo; 1994.

20. Denckla MB, Rudel RG. Rapid automatized naming of pictured objects, colors, letters and numbers by normal children. Cortex. 1974;10(2):186-202.

21. Dowell RC, Cowan RS. Evaluation of benefit: infants and children. In: Clark GM, Cowan RS, Dowell RC editors. Cochlear implantation for infants and children. San Diego: Singular; 1997. p. 205-22.

22. Mukari SZ, Ling LN, Ghani HA. Educational performance of pediatric cochlear implant recipients in mainstream classes. Int J Pediatr Otorhinolaryngol. 2007;71(2):231-40.

23. Marschark M, Rhoten C, Fabich M. Effects of cochlear implants on children's reading and academic achievement. J Deaf Stud Deaf Educ. 2007;12(3):269-82.

24. Pinotti KJ, Boscolo CC. A dramatização como estratégia da linguagem escrita para o deficiente auditivo. Rev Bras Educ Espec. 2008;14(1):121-40.

25. Connor CM, Zwolan TA. Examining multiple sources of influence on the reading comprehension skills of children who use cochlear implants. J Speech Lang Hear Res. 2004;47(3):509-26.

26. Geers A, Tobey E, Moog J, Brenner C. Longterm outcomes of cochlear implantation in the preschool years: from elementary grades to high school. Int J Audiol. 2008;47(sup.2):21-30.

27. Capellini S A, Tonelotto JMF, Ciasca SM. Medidas de desempenho escolar: avaliação formal e opinião de professores. Estud Psicol (Campinas). 2004;21(2):79-90.

28. Santos PL, Graminha SS. Problemas emocionais e comportamentais associados ao baixo rendimento acadêmico. Estud Psicol. (Natal). 2006:11(1):101-9.

29. Capellini AS, Ferreira TL, Salgado CA, Ciasca SM. Desempenho de escolares bons leitores, com dislexia e com transtorno do déficit de atenção e hiperatividade em nomeação automática rápida. Rev Soc Bras Fonoaudiol. 2007;12(2):114-9.

30. Stith JL, Drasgow E. Including children with cochlear implants in general education elementary classrooms. Teaching Exceptional Children Plus [serial on the internet]. 2005 Sept [cited 2010 July 16]; 2(1): [about 13 p.]. Available from: http:// escholarship.bc.edu/cgi/viewcontent.cgi?article $=10$ 08\&context=education/tecplus 
31. Ferreira TL, Capellini SA, Ciasca SM, Tonelotto JMF. Desempenho de escolares leitores proficientes no teste de nomeação automatizada rápida RAN. Temas Desenvolv. 2003; 12(69):26-32.

32. Swanson HL, Howard CB, Saez L. Do different components of working memory underlie different subgroups of reading disabilities? J Learn Disab. 2006; 39(3):252-69.

33. Gathercole SE, Baddeley AD. Working memory and language. Hove: Lawrence Erlbaum; 1995.

34. Mousinho R, Correa J. Habilidades linguísticocognitivas em leitores e não leitores. Pró-Fono. 2009;21(2):113-8.

http://dx.doi.org/10.1590/S1516-18462012005000059

RECEBIDO EM: 09/12/2010

ACEITO EM: 25/11/2011

Endereço para correspondência:

Ana Beatriz Sacomano Montassier Pinheiro

Rua São Gonçalo, 6-73 apto. 122 - Vila Altinópolis

Bauru/SP

CEP: $17012-170$

E-mail: anabeatriz.psico@gmail.com 